\title{
Nematode Interactions on Beetle Hosts Indicate a Role of Mouth-Form Plasticity in Resource Competition
}

\author{
Tess Renahan and Ralf J. Sommer* \\ Department for Integrative Evolutionary Biology, Max Planck Institute for Developmental Biology, Tübingen, Germany
}

OPEN ACCESS

Edited by:

Angelika Stollewerk,

Queen Mary University of London, United Kingdom

Reviewed by:

Alessandro Minelli,

University of Padua, Italy

Pedro Martinez,

University of Barcelona, Spain

${ }^{*}$ Correspondence:

Ralf J. Sommer

ralf.sommer@tuebingen.mpg.de

Specialty section:

This article was submitted to Evolutionary Developmental Biology, a section of the journal

Frontiers in Ecology and Evolution

Received: 03 August 2021

Accepted: 21 September 2021

Published: 14 October 2021

Citation:

Renahan $T$ and Sommer $R$ (2021) Nematode Interactions on

Beetle Hosts Indicate a Role of Mouth-Form Plasticity in Resource

Competition.

Front. Ecol. Evol. 9:752695. doi: 10.3389/fevo.2021.752695
Competition is rampant across kingdoms, arising over potential mates, food resources, and space availability. When faced with opponents, phenotypic plasticity proffers organisms indispensable advantageous strategies to outcompete rivals. This tactic is especially crucial on decaying insect hosts as myriad microbes and numerous nematodes struggle to establish thriving populations and ensure resource availability for future generations. Scarab beetles and their associated nematode symbionts on La Réunion Island have provided exceptional systems to study complicated crossphylum interactions in soil, and recently we have identified a previously unexplored beetle host, Gymnogaster bupthalma, to be reliably co-infested with diplogastrids Pristionchus mayeri and Acrostichus spp. These nematodes maintain the capacity to plastically respond to environmental conditions by developing disparate mouth forms, a strict bacterial-feeding morph or an omnivorous morph that enables predation on other nematodes. In addition, under stressful settings these worms can enter an arrested development stage called dauer, non-feeding dispersal larvae that resume development into reproducing adults when conditions improve. By investigating this beetle-nematode system in a natural context, we uncovered a novel Pristionchus strategy, wherein dauer dispersal from the carcass is gradual and a reproducing population is sustained. Remarkably, usually preferential-bacterial morph $P$. mayeri develop as predators in populations dense with competitors.

Keywords: phenotypic plasticity, nematode, Pristionchus, scarab beetle, competition, Acrostichus

\section{INTRODUCTION}

"The smallest Worme will turne, being trodden on".

William Shakespeare, 1951.

In the infamous historical composition Henry VI, Lord Clifford accurately assessed microscopic worms' capacities for turning to alternative forms when trodden on by competitors (Shakespeare, 2001). Indeed, phenotypic plasticity provides an imperative beneficial strategy in the face of competition and is employed by copious creatures, both flora and fauna (Miner et al., 2005; Stomp et al., 2008; Turcotte and Levine, 2016). Tadpoles of the spadefoot toad genus Spea can develop a carnivorous morph that serves as an alternative to the typical omnivorous morph under specific environmental conditions, allowing for consumption of its competitors (Pfennig and Murphy, 2002). Plants exhibit a plethora of plastic phenotypes in response to competition, including stem elongation and modified flowering time when overshadowed by opponents restricting shade, as comprehensively exemplified using 
model Arabidopsis thaliana (Callahan and Pigliucci, 2002; Donohue, 2003). When resource competition limits dung beetle Onthophagus taurus's favorite snack (due to increasing density), the insect can plastically respond in morphology, behavior, and life history traits (Macagno et al., 2016; Casasa and Moczek, 2018). However, beetles are not only prone to competition and the associated plastic responses, but also serve as the underground battlefield for competition among other organisms.

Competition among nematodes on insect cadavers is widespread and the conditions motley, depending on assorted factors including nematode species present, bacterial composition, and external environmental influences that may alter the already fluctuating settings (Dillman et al., 2012; Ali et al., 2013; Bertoloni Meli and Bashey, 2018; Blanco-Pérez et al., 2019; Renahan et al., 2021). The fight for food may be restricted to interspecies combat, but can also include conflict within species (Koppenhöfer et al., 1995; O'Callaghan et al., 2014). Advantages are largely species-dependent, and often as conspicuous as archetypal dietary range and developmental time, though more intricate pros may reflect worms' plastic capacities to expand nutritional sources and modify growth trajectories. Entomopathogenic nematodes (EPNs), obligate and occasional facultative parasites of insects, employ various strategies to dominate after inducing host death utilizing symbiotic bacteria (Burnell and Stock, 2000; Campos-Herrera et al., 2012). While some are quick to develop and populate the carcass, other EPNs rely on significantly larger initial populations; these strategies have been observed to reflect reproductive modes of the worms, with hermaphroditic EPNs depending on quick colonization and gonochoristic on original density (Duncan et al., 2003; CamposHerrera et al., 2015). To evade highly dense and competitive settings, a critical plastic property conserved across nematodes, an arrested developmental stage, is relied on. Named "infective juvenile" (IJ) in pathogenic worms and "dauer" in others, this alternative developmental pathway is often the dispersal stage; intraspecific density can result in continued dauer persistence (Sommer and Ogawa, 2011; Nermut' et al., 2012; Artyukhin et al., 2013) and on crowded insect cadavers, worms enter IJ when food has been depleted in search of a new host (Koppenhöfer et al., 1997; Rolston et al., 2006).

Necromenic model organism Pristionchus pacificus readily enters dauer under unfavorable environmental conditions and in response to competition (Mayer and Sommer, 2011; Bose et al., 2014). This valuable capacity is especially vital on insect carcasses, on which P. pacificus is often found (Herrmann et al., 2006, 2007). In addition to dauer, $P$. pacificus, along with a hodgepodge of diplogastrids, maintains mouth-form phenotypic plasticity (Von Lieven and Sudhaus, 2000; Kiontke and Fitch, 2010; Ragsdale et al., 2013), in which either a strict bacterial-feeding morph, stenostomatous (St) (Figure 1D), or an omnivorous morph that enables predation on other nematodes, eurystomatous $(\mathrm{Eu})$ (Figure 1C), is developed (Sudhaus, 2010; Susoy and Sommer, 2016). The combination of these two polyphenisms has been investigated both in laboratory and ecological contexts, utilizing the reliable association of $P$. pacificus with scarab beetle Oryctes borbonicus on La Réunion Island (Bento et al., 2010; Meyer et al., 2017; Renahan et al., 2021). La Réunion Island in the Indian
Ocean is home to a mélange of beetles associated with numerous nematodes (Herrmann et al., 2010), including the thoroughly studied O. borbonicus and P. pacificus (Meyer et al., 2017; Renahan et al., 2021). Nematode infestation rates vary among insects, though nearly $100 \%$ of O. borbonicus hosts P. pacificus, allowing for rare ecological experiments and deep dives into the evolutionary history of the worm (Morgan et al., 2012; McGaughran et al., 2016). While O. borbonicus and P. pacificus have served as an exceptional system to both study host-microbe interactions and dig into the ecology of a well-established model organism, the dearth of co-infestations of the beetle host with more than one nematode species has made competition studies in a natural context lacking.

Fortunately, we have recently discovered endemic cockchafer Gymnogaster bupthalma in abundance (Figure 1A), even though this beetle was previously assumed to be rare (see section "Materials and Methods" for details). Specifically, there were fewer than 15 specimens acquired between 1851 and 2018 (Lacroix, 1979; Gomy et al., 2017; Max Planck field work 20082018). Excitingly, as we ventured into formerly unexplored island territory, all three of our collection trips since 2019 resulted in copious G. bupthalma sightings, allowing for capture of enough specimen to study without disruption of the local population. Adult G. bupthalma is host to diplogastrids Pristionchus mayeri (Figures 1C,D) and species of the genus Acrostichus (Figure 1B), both capable of the mouth-form dimorphism (the latter depending on the species) (Giblin and Kaya, 1984; Kanzaki et al., 2013). Some beetle individuals only harbor one species, though there is no paucity of co-infestation with both nematodes; we utilized this naturally existing competition arena to investigate how these two nematodes fight for resources.

Globally found $P$. mayeri is strongly preferentially St (Figure 1D), even maintaining the bacterial-feeding morph under conventional Eu-inducing conditions, as developed and determined using several Pristionchus species (Wilecki et al., 2015; Werner et al., 2017). Since domesticated strains in a laboratory environment may not employ use of this discrete polyphenism, we sought to investigate how $P$. mayeri behaves when faced with competition in a natural setting, and if it utilizes its capacity for phenotypic plasticity. We collected and subsequently decapitated adult G. bupthalma (initiating nematode emergence), and tracked the succession and dynamics of the two nematode genera over 3 months post-beetle death. We uncovered a new Pristionchus dauer strategy, in which the larvae disperse gradually while maintaining a population on the carcass. In addition, we pinpoint the vital role of mouth-form plasticity in resource competition, wherein a predatory morph proliferates in environments dense with competitors (Figure 1C).

\section{MATERIALS AND METHODS}

\section{Beetle Collection and Cage Set-Up}

Male G. bupthalma were collected on La Réunion Island in January 2021 (Parc Nationale permit DIR-I-2020-280 and Nagoya number ABSCH-IRCC-FR-254969-1), when male adults are above ground for mate finding at night. Specimen were captured 

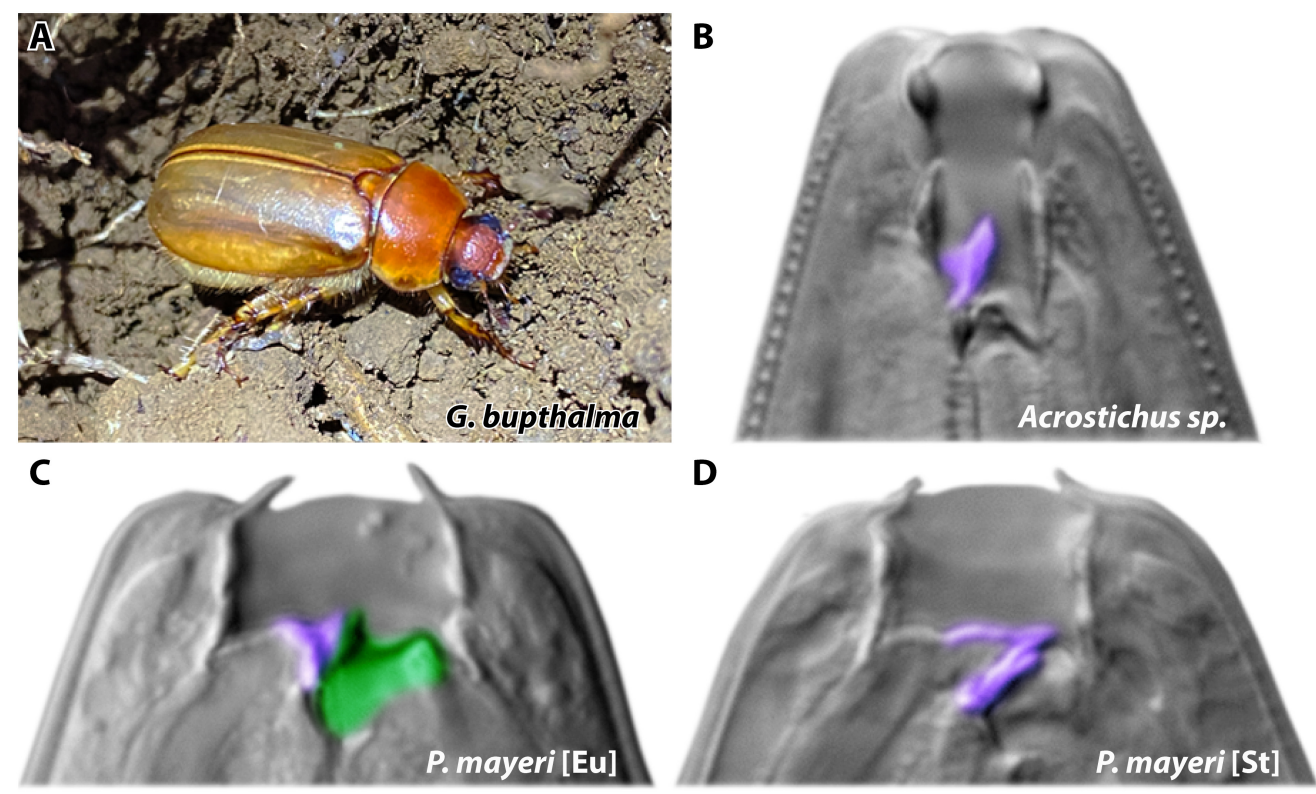

FIGURE 1 | Host beetle and mouth forms of symbiont nematodes. (A) Adult male beetle Gymnogaster bupthalma on La Réunion Island. Photo by Christian Weiler. (B-D) Dorsal tooth marked in purple, subventral in green. (B) Single-toothed Acrostichus is strictly bacterial-feeding. P. mayeri develops either (C) the wide predatory Eu morph with both the dorsal and subventral teeth or (D) the strict bacterial-feeding St morph with only the dorsal tooth.

by searching among trees post-dusk with headlights and then placed in $50 \mathrm{~mL}$ falcon tubes (5 per tube), with holes punctured in the falcon lids for aeration and wet towels added for moisture. The beetles were then flown to our lab in Tübingen, Germany for processing. Beetles were decapitated before being placed in autoclaved standard gardening soil (Einheitserde, Classic Profisubstrat) in $15 \times 5 \mathrm{~cm}$ cages made from $0.5 \mathrm{~mm}$ mesh. Cages were kept at $17^{\circ} \mathrm{C}$ and were watered to imitate the environment on the island. These methods are consistent with previous experiments establishing this set-up (Renahan et al., 2021). Eight cages were processed at each time point $(t=0,1$, and 4 days, 1, 2, 4, 6, 8, 10, and 12 weeks).

\section{Nematode Isolation, Screening, and Identification}

At the appropriate time points, the cages were disassembled, the beetle carcasses moved to nematode growth medium (NGM) plates, and the soil to Baermann funnels to isolate the nematodes (Viglierchio and Schmitt, 1983). Worms were screened for mouth form and screened based on morphology to identify species, then singled and later sequenced for further species verification. DNA was extracted using a lysis solution, followed by a polymerase chain reaction (PCR) to amplify a $1 \mathrm{~kb}$ region of the small subunit (SSU) rRNA gene using the primers SSU18A (5'-AAAGATTAAGCCATGCATG$\left.3^{\prime}\right)$ and SSU26R (5'CATTCTTGGCAAATGCTTTCG-3') (Blaxter et al., 1998). Sanger sequencing was carried out by GENEWIZ in Leipzig using sequencing primer SSU9R (5'AGCTGGAATtACCGCGGCTG-3'). SeqMan Pro was used to trim sequencing chromatograms, and NCBI reference database to blast for taxonomic identification. Unfortunately, due to limited data availability, we were not able to confidently determine the Acrostichus species present, as BLAST hits were consistently tied between A. nudicapitatus and A. halicti. Thus, potentially two, or even more unidentified, Acrostichus species are present on the carcass, but we treat these two as one species throughout the project.

\section{Competition Assays and Mouth-Form Phenotyping}

Singled worms from carcasses were left on NGM agar plates seeded with OP50 Escherichia coli to repopulate. The subsequent generations quickly entered dauer as the population density increased (more readily than domesticated lab strains tend to), and as worms are typically found in the dauer stage on live hosts (Herrmann et al., 2010; Ragsdale et al., 2015), these dauer worms were then used for competition assays. Worms were washed from plates and dauers isolated using $1 \%$ sodium dodecyl sulfate, then subsequently washed with M9 (Cassada and Russell, 1975). Dauers of the same species used as competitors against each other were first stained either CellTracker Green BODIPY (Thermo Fisher) or neutral red (Thomas and Lana, 2008) to differentiate the two populations during phenotyping, as established in Werner et al. (2018). The competition assays were composed of the following combinations: P. mayeri vs. Acrostichus, P. mayeri vs. P. mayeri from different carcasses, and $P$. mayeri vs. $P$. mayeri of the same strain. The same number of dauers for each competitor were added to NGM agar plates seeded with OP50 E. coli (to induce dauer exit, as on a carcass when microbes bloom), ranging from 10 dauers of each 
competitor to 250 dauers of each competitor. As control, dauers of each strain were isolated and grown on seeded plates at the same density as the total number of worms on an assay plate (e.g., in an assay of 250 P. mayeri strain 1 vs. 250 P. mayeri strain 2, control plates of 500 P. mayeri strain 1 and 500 P. mayeri strain 2 were tracked). Once the worms had developed into adults, they were mouth-form phenotyped using differential interference contrast (DIC) on a Zeiss Axioskop.

\section{RESULTS}

\section{Gymnogaster bupthalma Is Frequently Co-infested With Two Different Nematode Genera}

To determine nematode occurrence on G. bupthalma, we screened eight beetles per time point and found nearly each carcass was infested with at least one nematode, $P$. mayeri or Acrostichus. Almost a third of the carcasses (23 out of 80 beetles, $28.75 \%$ ) were infested with both nematodes (Figure 2A). Time of worm emergence from the carcasses varied among the time points: at $t=0$, decapitated carcasses were immediately plated on NGM agar, and nematodes emerged from 1 to 2 weeks after. This was consistently seen among the first four time points $(t=0$, $t=1$ day, $t=4$ days, and $t=1$ week), but by $t=2$ weeks, nematodes left the carcasses within 5 days. Nematodes in the surrounding soil were consistently observed at $t=2$ weeks and later ( $t=4$ weeks, $t=6$ weeks, $t=8$ weeks, $t=10$ weeks, and $t=12$ weeks), with three replicates at $t=1$ week having few worms in the surrounding soil, and none in the earlier time points. Succession in the earlier time points were not distinguished $(t=0$, 1 , and 4 days), likely due to the duration required for worms to emerge; thus a couple days between these time points do not confer any conspicuous differences. In specimen infested with either a single species or with two, time of departure was independent of nematode type. Though, at earlier time points ( $t=0$ through $t=2$ weeks), in co-infested carcasses, Acrostichus was more prevalent in numbers of worms than $P$. mayeri, but the two equalized after 1 month on the carcasses. On the carcass, worms were found in both feeding mixed stages and the arrested development dauer stage (Figure 2B), independent of whether they were in co-inhabited space or singly lived. From $t=2$ to $t=6$ weeks there was a steep increase in the number of both dauers and feeding stages (from 30 dauers and 10 feeding stages at $t=2$ weeks to 160 dauers and 130 feeding stages at $t=6$ weeks). From then, both populations drop, and at $t=12$ weeks only 12 dauers and 1 feeding stage worm remain. In the surrounding soil, worms were exclusively in dauer, the dispersal stage.

\section{Succession of Pristionchus mayeri Mouth Form Over 3 Months}

We tracked the mouth forms of $P$. mayeri over the course of 3 months post-beetle death when co-infested with Acrostichus (Figure 3A). P. mayeri developed only the strict bacterial-feeding St morph at early time points ( $t=0-1$ week), when population densities on the carcasses are relatively low. But, at $t=2$ weeks

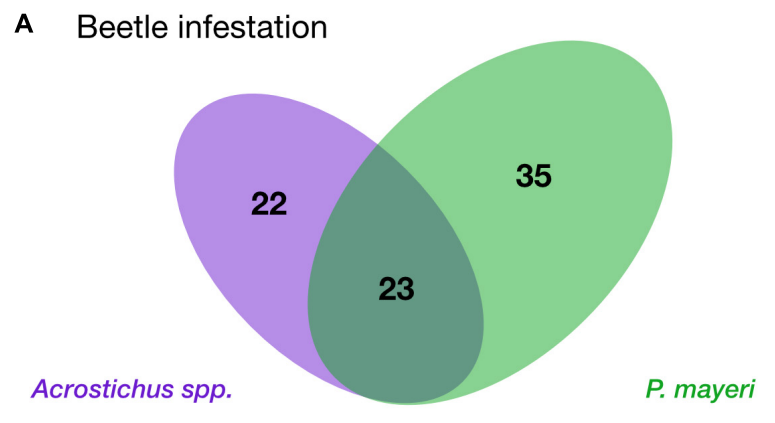

\section{B Nematode succession on beetle carcasses}

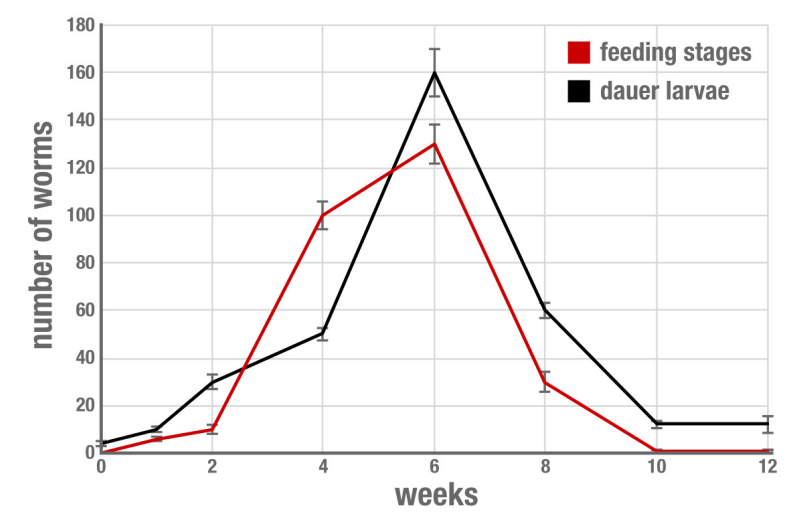

FIGURE 2 | Reliably co-infested G. bupthalma remains home to various stages of both P. mayeri and Acrostichus. (A) 80 adult G. bupthalma were investigated across 3 months. A total of 22 individuals harbored only Acrostichus spp. and 35 only P. mayeri. 23 individuals were infested with both Acrostichus and P. mayeri. (B) Nematode succession on the carcass over 3 months. The populations of both dauers (black) and feeding mixed stages (red) increase during the first 6 weeks, reaching a peak at 6 weeks (160 dauers and 130 feeding stages), and then decrease until the population is almost depleted at 12 weeks (12 dauers and 1 feeding stage). $N=8$ beetles per time point, average number shown. Error bars are standard error of the mean.

as population increases, $P$. mayeri starts also developing into the predatory morph, with $36.5 \%$ being Eu. The number of Eu animals increases with some fluctuation $(38 \%$ at $t=2$ weeks, $84 \%$ at $t=4$ weeks, $62 \%$ at $t=6$ weeks), and reaches $100 \% \mathrm{Eu}$ at $t=8$ weeks. While the number of worms reaches its peak at $t=6$ weeks (160 dauers, 130 feeding stages), at the dip during $t=8$ weeks (60 dauers and 30 feeding stages), P. mayeri still employs the predatory morph at $100 \%$ frequency. Though, at $t=10$ and 12 weeks there are few worms on the carcasses, and the predatory prevalence drops to $20 \%$ and $17 \%$, respectively. Thus, as population density increases alongside the presence of competitors, more $P$. mayeri become predatory.

\section{Competition Experiments Reveal Cross-Genera Induction of the Predatory Morph}

To determine if the increase in predatory mouth forms in $P$. mayeri indeed reflects the presence of competition, we 
A P. mayeri morph succession on carcasses [Eu/St]

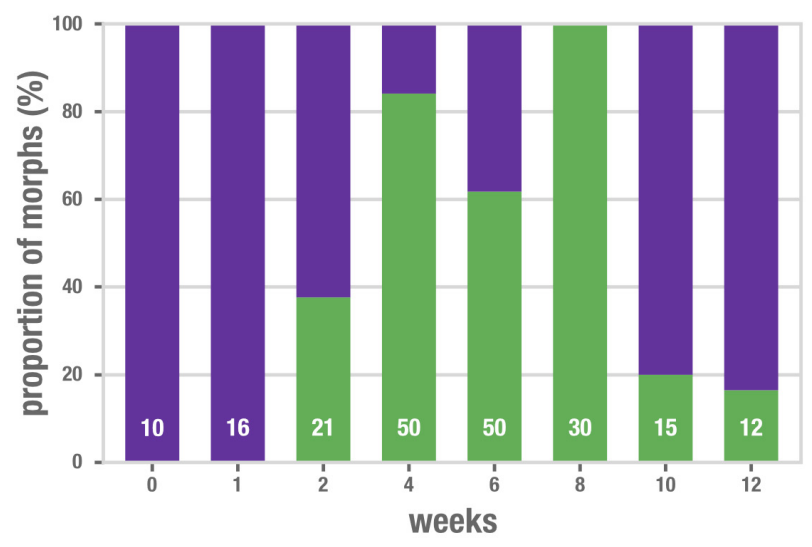

C P. mayeri versus $P$. mayeri $[\mathrm{Eu} / \mathrm{St}]$

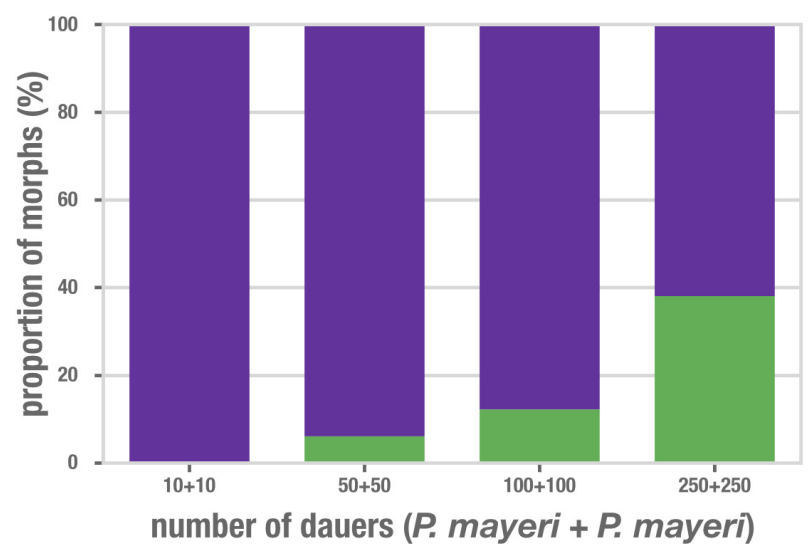

B P. mayeri versus Acrostichus spp. [Eu/St]

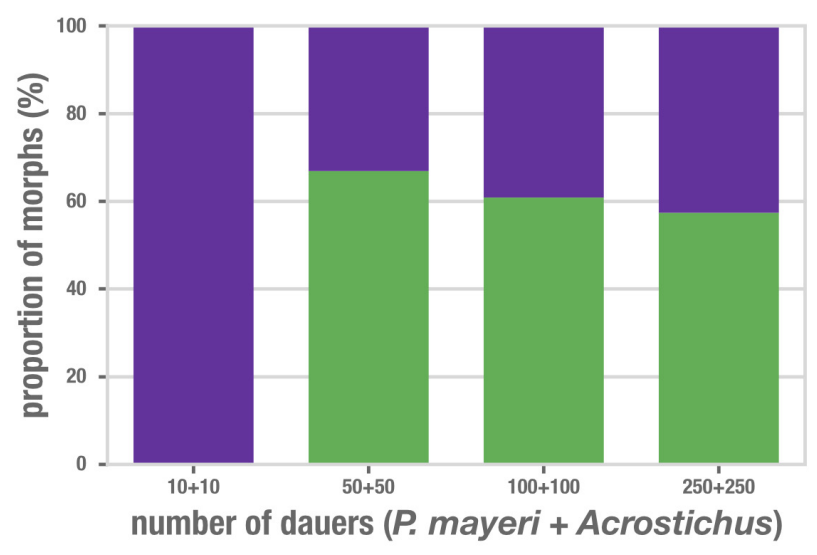

D P. mayeri $[\mathrm{Eu} / \mathrm{St}]$

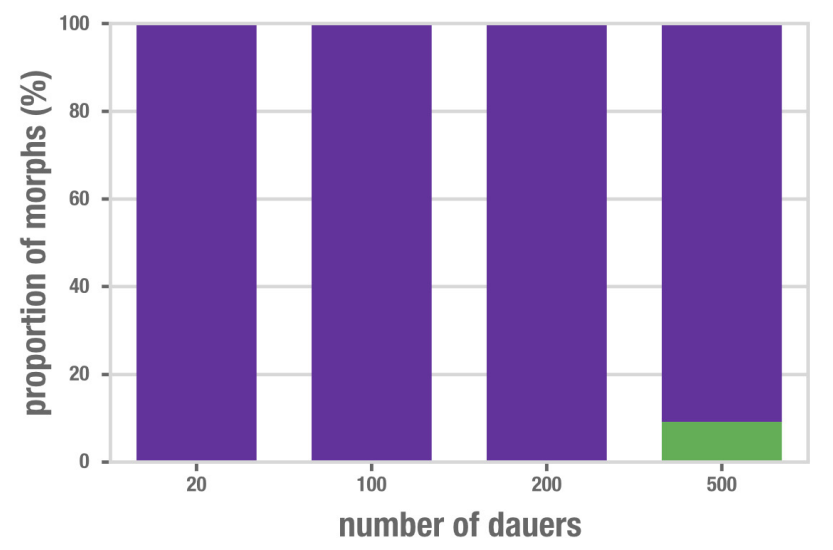

FIGURE 3 | Pristionchus mayeri develops the predatory morph in populations dense with competitors. (A) Succession of mouth-form ratios of $P$. mayeri on beetle carcasses co-infested with Acrostichus. $\mathrm{N}$ is shown in white for each time point; the range reflects the variability of number of individuals present as displayed in Figure 2B. The number of predators increases as the density on the carcass increases ( $t=2-8$ weeks). Though, when the population is low at $t=0,1$, 10 , and 12 weeks, the prevalence of predators decreases. Competition assays (B-D) using dauer lines from the worms observed in panel (A). (B) P. mayeri vs. Acrostichus in increasing density of dauers shows an increase in the predatory morph. Fisher's exact test on summed Eu counts against the respective dauer density as shown in panel (D): $p<0.05$ for densities 50:50, 100:100, and 250:250. $N=100$ per bar, 10 strains of each competitor in various combinations. (C) $P$. mayeri vs. $P$. mayeri from different carcasses in increasing density of dauers. Fisher's exact test on summed Eu counts against the respective dauer density as shown in panel (D): $p<0.05$ for densities 100:100 and 250:250. $N=100$ per bar, 10 strains of each competitor in various combinations. (D) Control $P$. mayeri assays in which singled strains were grown at the same densities as in the competition assays of (B,D). $N=100$ per bar, 10 strains each.

maintained lines of both genera from multiple carcasses and time points, and induced dauer simply by allowing the NGM agar plates to become overcrowded. While we isolated and grew lines of $P$. mayeri that were Eu on the carcass, once on plates these strains became St. Dauers were isolated, stained, and mixed on fresh plates with food in the following combinations: P. mayeri vs. Acrostichus, $P$. mayeri vs. $P$. mayeri from different carcasses, and $P$. mayeri against itself. As few as 10 dauers of each strain were added to reflect the low number of worms at early time points, and as many as 250 dauers of each strain to reflect the high population densities at $t=4$ and 6 weeks seen in Figure 2B. $P$. mayeri maintained the St morph in all assays with $10+10$ dauers, regardless of whether the competition was Acrostichus, another P. mayeri strain, or itself (Figures 3B-D). This is consistent with the lack of predatory $P$. mayeri observed in early carcass time points when nematode density is rather low. Though, once the population in the competition assays increased to 50 vs. 50, two-thirds (66\%) of P. mayeri developed the predatory morph when the competition was Acrostichus (Figure 3B). The same $P$. mayeri strain in competition with itself exclusively exhibited the St morph (Figure 3D). Though, when $50 \mathrm{P}$. mayeri were paired with $50 \mathrm{P}$. mayeri of a different strain, 6\% became Eu (Figure 3C), while those strains against themselves remained St. This trend continues as the number of dauers increases: $P$. mayeri vs. Acrostichus results in over $50 \% \mathrm{Eu}$ (Figure 3B) and even $P$. mayeri vs. P. mayeri become $40 \% \mathrm{Eu}$ when the density is 250 vs. 250 (Figure 3C). Isolated P. mayeri, however, even in high density (500), fail to produce many Eu, with the highest being just shy of $10 \%$ (Figure 3D). Thus, $P$. mayeri is prone to develop the predatory morph in competitive 


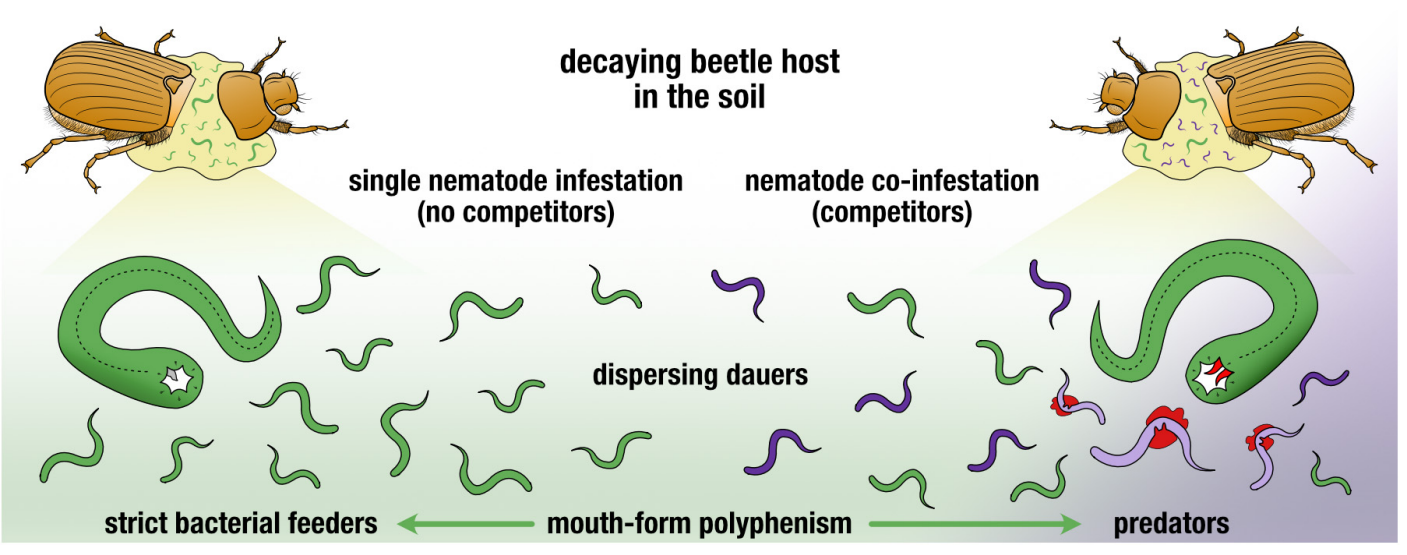

FIGURE 4 | Graphical summary of two nematode plastic responses in face of resource competition post-beetle death. Dauers continuously disperse and $P$. mayeri develops as predators when co-infesting the host with Acrostichus.

and dense environments, especially when the competitor is of another genus (Acrostichus) and even when the competitor is of the same species but another line. The mouth-form pattern observed in the competition assays mirror that of the observed mouth-form pattern in the natural carcass setting: as competitor density increases, so does the occurrence of the predatory morph.

\section{DISCUSSION}

For the first time, we explored the dynamics of co-occurring nematodes on the previously uninvestigated beetle G. bupthalma. We found the adult beetle to be reliably infected with at least one nematode genus, Pristionchus or Acrostichus, and often coinfested with both, allowing for unique studies of nematode competition in a natural setting. By decapitating beetles and thus inducing nematode emergence, we were able to track the succession and plastic responses of worms over 3 months. Strikingly, we found that $P$. mayeri utilizes its capacity to develop the predatory $\mathrm{Eu}$ morph when faced with dense competition. This trend toward predation was verified using freshly isolated P. mayeri lines in competition assays with also newly cultured strains of Acrostichus, revealing the vital role of mouth-form plasticity in resource competition. Our combined field and laboratory study system resulted in three major conclusions.

First, we found a novel dispersal strategy of Pristionchus dauer larvae. As we tracked nematode succession on the carcasses, we expected a pattern of feeding stages and the arrested developmental and dispersal stage, dauer. Surprisingly, we did not observe an inverse presence/absence of feeding stages versus dauer; worms consistently developed via both pathways (Figure 2B). Though, this is in contrast to previous studies on $O$. borbonicus and $P$. pacificus, in which feeding stages were in low abundance while dauers were numerous, and vice versa; this was found to reflect bacterial load, and thus food availability (Renahan et al., 2021). Perhaps since P. pacificus is often exclusively the solitary diplogastrid on its host, its dauer plasticity response is weighted toward resource abundance, while on G. bupthalma, the two nematode genera must also factor in the occurrence of the other worm. While $P$. pacificus displays a biphasic boom and bust strategy with two major dispersal events from the carcass, P. mayeri and Acrostichus dauers steadily and gradually disperse from the carcass, while also sustaining a feeding and reproducing population in order to maintain a competitive advantage, and do so throughout the first 2 months until food is depleted. Thus, on hosts in which two diplogastrids frequently co-occur, the worm dispersal strategy differs from that of worms that infest hosts often solitarily. These findings add to the complexity of behavioral strategies of dauer-associated traits.

Second, nematode interactions indicate a role of mouthform plasticity in resource competition. In addition to entering the dauer stage to disperse, these nematodes retain the plastic ability to develop either a strict bacterial-feeding (St) morph or the omnivorous $(\mathrm{Eu})$ morph that allows for predation on nematode larvae. Domesticated strains of P. mayeri tend toward the St morph, rarely developing Eu, even when Eu induction is attempted by established methods (Wilecki et al., 2015; Werner et al., 2017). Intriguingly, P. mayeri is preferentially St when few worms populate the carcass, but as density increases temporally, more and more P. mayeri become predators (Figure 3A). While certain species of Acrostichus have also displayed the polyphenism, dimorphism was not observed during this perhaps limited exploration. Though these observations are consistent with recent studies detailing the association of reproductive mode and mouth form, in which hermaphrodites display predatory tendencies to incite competition, while gonochoristic species are preferentially bacterivorous to deter conflict between parents and offspring (Lightfoot et al., 2021). Accordingly, hermaphroditic P. mayeri becomes predatory without risk of compromising its own kin, while Acrostichus remains non-predatory and avoids potential kin consumption.

Third, we confirmed that $P$. mayeri's increase in the predatory morph is a consequence of higher competitor density by conducting assays pairing worms against each other. Indeed, P. mayeri becomes more predatory with higher abundances of Acrostichus (Figure 3B), and to a more muted extent, with higher 
abundances of other P. mayeri strains (Figure 3C). Though, the occurrence of predators in these assays is not as high as observed in the natural setting of the beetle carcass. Myriad of factors can explain this aberration, as copious affairs are ongoing on the carcass, both seemingly detectable and not, and that we did not fully replicate or pinpoint in our assays. Mainly, the bacteria present on the decaying carcass may play roles both in worm developmental stages and thus dispersal, and mouth form tendencies. Bacterial influence on nematode polyphenisms is well-explored, with several studies demonstrating microbial impact on dauer induction, Eu formation, and predatory rates (Samuel et al., 2016; Akduman et al., 2018, 2020; Bubrig et al., 2020).

Furthermore, nematodes chemically communicate via small molecules, nematode-derived modular metabolites (NDMMs), that regulate various behaviors and trigger plastic responses, including dauer entry and exit and mouth form (Bento et al., 2010; Bose et al., 2012). In particular, the complex ascaroside dasc\#1 induces the predatory morph in P. pacificus in a density and age-specific manner (Werner et al., 2018). P. mayeri also produces this pheromone, though not to the same extent that $P$. pacificus does, while it is undetected in Acrostichus (Dong et al., 2020). The presence of a known Eu-inducing molecule in P. mayeri and its absence in Acrostichus may explain the development of predators in the former genus and lack of in the latter. Though, as the abundance of dasc\#1 is relatively low in $P$. mayeri, and Eu worms are not observed in dense populations of singled P. mayeri strains (Figure 3D), it is clear that other factors maintain vital roles in influencing development of the predatory morph.

All together, we have demonstrated a plastic response of a preferentially bacterivorous species to become predatory when faced with competition in a natural setting (Figure 4). While population density and pheromone communication explain aspects of this trend, supplementary exploration of the prominent

\section{REFERENCES}

Akduman, N., Lightfoot, J. W., Röseler, W., Witte, H., Lo, W. S., Rödelsperger, C., et al. (2020). Bacterial vitamin B 12 production enhances nematode predatory behavior. ISME J. 14, 1494-1507. doi: 10.1038/s41396-020-0626-2

Akduman, N., Rödelsperger, C., and Sommer, R. J. (2018). Culture-based analysis of Pristionchus-associated microbiota from beetles and figs for studying nematode-bacterial interactions. PLoS One 13:e0198018. doi: 10.1371/journal. pone.0198018

Ali, J. G., Campos-Herrera, R., Alborn, H. T., Duncan, L. W., and Stelinski, L. L. (2013). Sending mixed messages: a trophic cascade produced by a belowground herbivore-induced cue. J. Chem. Ecol. 39, 1140-1147. doi: 10.1007/s10886-0130332-x

Artyukhin, A. B., Schroeder, F. C., and Avery, L. (2013). Density dependence in Caenorhabditis larval starvation. Sci. Rep. 3, 1-7. doi: 10.1038/srep02777

Bento, G., Ogawa, A., and Sommer, R. J. (2010). Co-option of the hormonesignalling module dafachronic acid-DAF-12 in nematode evolution. Nature 466, 494-497. doi: 10.1038/nature09164

Bertoloni Meli, S., and Bashey, F. (2018). Trade-off between reproductive and anticompetitor abilities in an insect-parasitic nematode-bacteria symbiosis. Ecol. Evol. 8, 10847-10856. doi: 10.1002/ece3.4538

Blanco-Pérez, R., Bueno-Pallero, F. Á, Vicente-Díez, I., Marco-Mancebón, V. S., Pérez-Moreno, I., and Campos-Herrera, R. (2019). Scavenging behavior and carcass microbiome may reveal further factors influencing competitive strategies. In addition, deeper investigating of the co-occurrence of these two nematode genera in other beetle life stages will shed light on the dynamics of the nematodes with its host, alongside the bacterial succession on the beetle developmental stages. Conclusively, we have established that when $P$. mayeri is trodden on by competition, it turns predatory.

\section{DATA AVAILABILITY STATEMENT}

The original contributions presented in the study are included in the article/supplementary material, further inquiries can be directed to the corresponding author.

\section{AUTHOR CONTRIBUTIONS}

TR and RS conceived the study. TR conducted all experiments, analyzed all data, and wrote the manuscript with edits from RS. Both authors contributed to the article and approved the submitted version.

\section{FUNDING}

This work was funded by the Max Planck Society.

\section{ACKNOWLEDGMENTS}

We thank the La Réunion team, including Matthias Herrmann and Christian Weiler, for beetle collection and the beetle picture. We greatly appreciate Tobias Theska for discussions and illustration help.

interspecific competition decrease offspring fitness of the entomopathogenic nematode Steinernema feltiae. J. Invertebr. Pathol. 164, 5-15. doi: 10.1016/j.jip. 2019.04.002

Blaxter, M. L., De Ley, P., Garey, J. R., Liu, L. X., Scheldeman, P., Vierstraete, A., et al. (1998). A molecular evolutionary framework for the phylum Nematoda. Nature 392, 71-75. doi: 10.1038/32160

Bose, N., Meyer, J. M., Yim, J. J., Mayer, M. G., Markov, G. V., Ogawa, A., et al. (2014). Natural variation in dauer pheromone production and sensing supports intraspecific competition in nematodes. Curr. Biol. 24, 1536-1541. doi: 10.1016/j.cub.2014. 05.045

Bose, N., Ogawa, A., von Reuss, S. H., Yim, J. J., Ragsdale, E. J., Sommer, R. J., et al. (2012). Complex small-molecule architectures regulate phenotypic plasticity in a nematode. Angew. Chem 124, 12606-12611. doi: 10.1002/ange. 201206797

Bubrig, L. T., Sutton, J. M., and Fierst, J. L. (2020). Caenorhabditis elegans dauer recovery varies with worm-bacteria interactions. Ecol. Evol. 10, 9886-9895. doi: 10.1002/ece3.6646

Burnell, A., and Stock, S. P. (2000). Heterorhabditis, Steinernema and their bacterial symbionts-lethal pathogens of insects. Nematology 2, 31-42. doi: $10.1163 / 156854100508872$

Callahan, H. S., and Pigliucci, M. (2002). Shade-induced plasticity and its ecological significance in wild populations of Arabidopsis thaliana. Ecology 83, 1965-1980. 
Campos-Herrera, R., Barbercheck, M., Hoy, C. W., and Stock, S. P. (2012). Entomopathogenic nematodes as a model system for advancing the frontiers of ecology. J. Nematol. 44:162.

Campos-Herrera, R., Pùža, V., Jaffuel, G., Blanco-Pérez, R., Èepulytë-Rakauskienë, R., and Turlings, T. C. (2015). Unraveling the intraguild competition between Oscheius spp. nematodes and entomopathogenic nematodes: implications for their natural distribution in Swiss agricultural soils. J. Invertebr. Pathol. 132, 216-227. doi: 10.1016/j.jip.2015.10.007

Casasa, S., and Moczek, A. P. (2018). The role of ancestral phenotypic plasticity in evolutionary diversification: population density effects in horned beetles. Anim. Behav. 137, 53-61. doi: 10.1016/j.anbehav.2018.01.004

Cassada, R. C., and Russell, R. L. (1975). The dauerlarva, a post-embryonic developmental variant of the nematode Caenorhabditis elegans. Dev. Biol. 46, 326-342. doi: 10.1016/0012-1606(75)90109-8

Dillman, A. R., Chaston, J. M., Adams, B. J., Ciche, T. A., Goodrich-Blair, H., Stock, S. P., et al. (2012). An entomopathogenic nematode by any other name. PLoS Pathog. 8:e1002527. doi: 10.1371/journal.ppat.1002527

Dong, C., Weadick, C. J., Truffault, V., and Sommer, R. J. (2020). Convergent evolution of small molecule pheromones in Pristionchus nematodes. eLife 9:e55687. doi: 10.7554/eLife.55687

Donohue, K. (2003). Setting the stage: phenotypic plasticity as habitat selection. Int. J. Plant Sci. 164, S79-S92. doi: 10.1086/368397

Duncan, L. W., Dunn, D. C., Bague, G., and Nguyen, K. (2003). Competition between entomopathogenic and free-living bactivorous nematodes in larvae of the weevil Diaprepes abbreviatus. J. Nematol. 35:187.

Giblin, R. M., and Kaya, H. K. (1984). Aduncospiculum halicti n. gen., n. sp.(Diplogasterida: Diplogasteroididae), an associate of bees in the genus Halictus (Hymenoptera: Halictidae). Rev. Nématol. 7, 189-197.

Gomy, Y., Lemagnen, R., and Poussereau, J. (2017). Les Coléoptères de I'T̂l de La Réunion. London: Nhbs Ltd.

Herrmann, M., Kienle, S., Rochat, J., Mayer, W. E., and Sommer, R. J. (2010). Haplotype diversity of the nematode Pristionchus pacificus on Réunion in the Indian Ocean suggests multiple independent invasions. Biol. J. Linn. Soc. 100, 170-179. doi: 10.1111/j.1095-8312.2010.01410.x

Herrmann, M., Mayer, W. E., Hong, R. L., Kienle, S., Minasaki, R., and Sommer, R. J. (2007). The nematode Pristionchus pacificus (Nematoda: Diplogastridae) is associated with the oriental beetle Exomala orientalis (Coleoptera: Scarabaeidae) in Japan. Zool. Sci. 24, 883-889. doi: 10.2108/zsj.24. 883

Herrmann, M., Mayer, W. E., and Sommer, R. J. (2006). Nematodes of the genus Pristionchus are closely associated with scarab beetles and the Colorado potato beetle in Western Europe. Zoology 109, 96-108. doi: 10.1016/j.zool.2006.03.001

Kanzaki, N., Ragsdale, E. J., Herrmann, M., Susoy, V., and Sommer, R. J. (2013). Two androdioecious and one dioecious new species of Pristionchus (Nematoda: Diplogastridae): new reference points for the evolution of reproductive mode. J. Nematol. 45:172.

Kiontke, K., and Fitch, D. H. (2010). Phenotypic plasticity: different teeth for different feasts. Curr. Biol. 20, R710-R712. doi: 10.1016/j.cub.2010.07.009

Koppenhöfer, A. M., Baur, M. E., Stock, S. P., Choo, H. Y., Chinnasri, B., and Kaya, H. K. (1997). Survival of entomopathogenic nematodes within host cadavers in dry soil. Appl. Soil Ecol. 6, 231-240. doi: 10.1016/S0929-1393(97)00018-8

Koppenhöfer, A. M., Kaya, H. K., Shanmugam, S., and Wood, G. L. (1995). Interspecific competition between steinernematid nematodes within an insect host. J. Invertebr. Pathol. 66, 99-103. doi: 10.1006/jipa.1995.1070

Lacroix, M. (1979). Gymnogaster buphthalma Blanchard, espèce énigmatique de l'île de la Réunion (Coleoptera Melolonthidae). Rev. Franç. D'entomol. 1988, 25-29.

Lightfoot, J. W., Dardiry, M., Kalirad, A., Giaimo, S., Eberhardt, G., Witte, H., et al. (2021). Sex or cannibalism: polyphenism and kin recognition control social action strategies in nematodes. Sci. Adv. [Epub ahead of print].

Macagno, A. L., Moczek, A. P., and Pizzo, A. (2016). Rapid divergence of nesting depth and digging appendages among tunneling dung beetle populations and species. Am. Nat. 187, E143-E151. doi: 10.1086/ 685776

Mayer, M. G., and Sommer, R. J. (2011). Natural variation in Pristionchus pacificus dauer formation reveals cross-preference rather than self-preference of nematode dauer pheromones. Proc. R. Soc. B Biol. Sci. 278, 2784-2790. doi: $10.1098 /$ rspb. 2010.2760
McGaughran, A., Rödelsperger, C., Grimm, D. G., Meyer, J. M., Moreno, E., Morgan, K., et al. (2016). Genomic profiles of diversification and genotypephenotype association in island nematode lineages. Mol. Biol. Evol. 33, 22572272. doi: 10.1093/molbev/msw093

Meyer, J. M., Baskaran, P., Quast, C., Susoy, V., Rödelsperger, C., Glöckner, F. O., et al. (2017). Succession and dynamics of Pristionchus nematodes and their microbiome during decomposition of Oryctes borbonicus on La Réunion Island. Environ. Microbiol. 19, 1476-1489. doi: 10.1111/1462-2920. 13697

Miner, B. G., Sultan, S. E., Morgan, S. G., Padilla, D. K., and Relyea, R. A. (2005). Ecological consequences of phenotypic plasticity. Trends Ecol. Evol. 20, 685-692. doi: 10.1016/j.tree.2005.08.002

Morgan, K., McGaughran, A., Villate, L., Herrmann, M., Witte, H., Bartelmes, G., et al. (2012). Multi locus analysis of Pristionchus pacificus on La Réunion Island reveals an evolutionary history shaped by multiple introductions, constrained dispersal events and rare out-crossing. Mol. Ecol. 21, 250-266. doi: 10.1111/j. 1365-294X.2011.05382.x

Nermut', J., Pùža, V., and Mráèek, Z. (2012). The effect of intraspecific competition on the development and quality of Phasmarhabditis hermaphrodita (Rhabditida: Rhabditidae). Biocontrol Sci. Technol. 22, 1389-1397. doi: 10.1080/ 09583157.2012.730604

O’Callaghan, K. M., Zenner, A. N., Hartley, C. J., and Griffin, C. T. (2014). Interference competition in entomopathogenic nematodes: male Steinernema kill members of their own and other species. Int. J. Parasitol. 44, 1009-1017. doi: 10.1016/j.ijpara.2014.07.004

Pfennig, D. W., and Murphy, P. J. (2002). How fluctuating competition and phenotypic plasticity mediate species divergence. Evolution 56, 1217-1228. doi: 10.1111/j.0014-3820.2002.tb01433.x

Ragsdale, E. J., Kanzaki, N., and Herrmann, M. (2015). Taxonomy and natural history: the genus Pristionchus. Pristionchus Pacificus 1, 77-120. doi: 10.1163/ 9789004260306_005

Ragsdale, E. J., Müller, M. R., Rödelsperger, C., and Sommer, R. J. (2013). A developmental switch coupled to the evolution of plasticity acts through a sulfatase. Cell 155, 922-933. doi: 10.1016/j.cell.2013. 09.054

Renahan, T., Lo, W. S., Werner, M. S., Rochat, J., Herrmann, M., and Sommer, R. J. (2021). Nematode biphasic 'boom and bust' dynamics are dependent on host bacterial load while linking dauer and mouth-form polyphenisms. Environ. Microbiol. [Epub ahead of print]. doi: 10.1111/1462-2920. 15438

Rolston, A. N., Griffin, C. T., and Downes, M. J. (2006). Emergence and dispersal patterns of two isolates of the entomopathogenic nematode Steinernema feltiae. J. Nematol. 38:221.

Samuel, B. S., Rowedder, H., Braendle, C., Félix, M. A., and Ruvkun, G. (2016). Caenorhabditis elegans responses to bacteria from its natural habitats. Proc. Natl. Acad. Sci. U.S.A. 113, E3941-E3949. doi: 10.1073/pnas.1607183113

Shakespeare, W. (2001). Henry VI.: Part Three. New York, NY: Oxford University Press, USA.

Sommer, R. J., and Ogawa, A. (2011). Hormone signaling and phenotypic plasticity in nematode development and evolution. Curr. Biol. 21, R758-R766. doi: 10. 1016/j.cub.2011.06.034

Stomp, M., van Dijk, M. A., van Overzee, H. M., Wortel, M. T., Sigon, C. A., Egas, M., et al. (2008). The timescale of phenotypic plasticity and its impact on competition in fluctuating environments. Am. Nat. 172, E169-E185. doi: $10.1086 / 591680$

Sudhaus, W. A. (2010). Preadaptive plateau in Rhabditida (Nematoda) allowed the repeated evolution of zooparasites, with an outlook on evolution of life cycles within Spiroascarida. Palaeodiversity. 3(Suppl.), 117-130.

Susoy, V., and Sommer, R. J. (2016). Stochastic and conditional regulation of nematode mouth-form dimorphisms. Front. Ecol. Evol. 4:23. doi: 10.3389/fevo. 2016.00023

Thomas, M. C., and Lana, P. D. (2008). Evaluation of vital stains for free-living marine nematodes. Brazil. J. Oceanogr. 56, 249-251.

Turcotte, M. M., and Levine, J. M. (2016). Phenotypic plasticity and species coexistence. Trends Ecol. Evol. 31, 803-813. doi: 10.1016/j.tree.2016.07.013

Viglierchio, D. R., and Schmitt, R. V. (1983). On the methodology of nematode extraction from field samples: baermann funnel modifications. J. Nematol. $15: 438$. 
Von Lieven, A. F., and Sudhaus, W. (2000). Comparative and functional morphology of the buccal cavity of Diplogastrina (Nematoda) and a first outline of the phylogeny of this taxon. J. Zool. Syst. Evol. Res. 38, 37-63. doi: 10.1046/j. 1439-0469.2000.381125.x

Werner, M. S., Claaßen, M. H., Renahan, T., Dardiry, M., and Sommer, R. J. (2018). Adult influence on juvenile phenotypes by stage-specific pheromone production. Iscience 10, 123-134. doi: 10.1016/j.isci.2018. 11.027

Werner, M. S., Sieriebriennikov, B., Loschko, T., Namdeo, S., Lenuzzi, M., Dardiry, M., et al. (2017). Environmental influence on Pristionchus pacificus mouth form through different culture methods. Sci. Rep. 7, 1-2. doi: 10.1038/s41598-01707455-7

Wilecki, M., Lightfoot, J. W., Susoy, V., and Sommer, R. J. (2015). Predatory feeding behaviour in Pristionchus nematodes is dependent on phenotypic plasticity and induced by serotonin. J. Exp. Biol. 218, 1306-1313. doi: 10.1242/jeb. 118620
Conflict of Interest: The authors declare that the research was conducted in the absence of any commercial or financial relationships that could be construed as a potential conflict of interest.

Publisher's Note: All claims expressed in this article are solely those of the authors and do not necessarily represent those of their affiliated organizations, or those of the publisher, the editors and the reviewers. Any product that may be evaluated in this article, or claim that may be made by its manufacturer, is not guaranteed or endorsed by the publisher.

Copyright $\odot 2021$ Renahan and Sommer. This is an open-access article distributed under the terms of the Creative Commons Attribution License (CC BY). The use, distribution or reproduction in other forums is permitted, provided the original author(s) and the copyright owner(s) are credited and that the original publication in this journal is cited, in accordance with accepted academic practice. No use, distribution or reproduction is permitted which does not comply with these terms. 